\title{
UML-based Radar Software Design
}

\section{Feng Juntao}

East China Research Institute of Electronic Engineering, Hefei, 230088

Keywords: UML modeling; radar software; database

\begin{abstract}
In view of the increasing complexity of radar software, this paper applies UML modeling technology to analyze the function and design of radar software. Based on requirements of radar software, it can be recognized that the system functions include remote control, parameter setting, track management, and device management. In order to help facilitate the development of the system, UML modeling class diagrams are used for corresponding functional design and database design. Through the above design, it is finally ready for radar software development.
\end{abstract}

\section{Introduction}

Radar is the use of radio methods to find targets and locate their space, usually for military and civilian purposes. System development is required for radar management. In [1], UML (Unified Modeling Language) unified modeling language was utilized to build a radar test system. The results show that using the UML modeling language can improve software development efficiency. The literature [2] uses the UML modeling language for radar software use case analysis, test analysis, and software development. The literature [3-4] uses Visual C+ language to carry on the system development, uses the UML modeling language to carry on the use case analysis in the early development, the system developed satisfies the battle platform demand. The literature [5] uses UML modeling technology to carry out message-driven event simulation. The results show that the improved system satisfies the system requirements. This article plans to use UML modeling technology to improve radar software development on the basis of predecessors.

\section{Functional requirements of System}

The radar management system includes the following functions: radar remote control, parameter setting, track management, equipment management. In radar remote control, radar equipment control is mainly carried out through the network. Parameter setting is used to manage the corresponding parameters in radar. Track management radar track. Equipment management is used to manage the equipment involved in radar.

Remote control is mainly through the TCP protocol for radar control. The functions listed in this function include remote shutdown, remote restart, and remote standby. Figure 1 shows the remote control use case diagram.

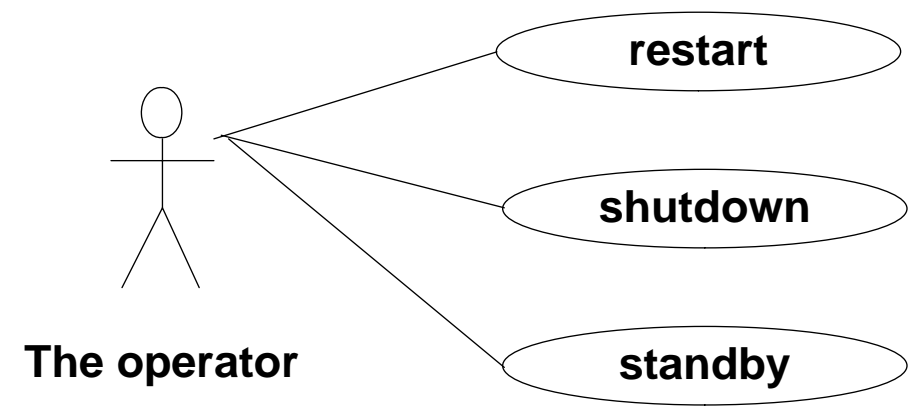

Figure 1 Remote control use case diagram 


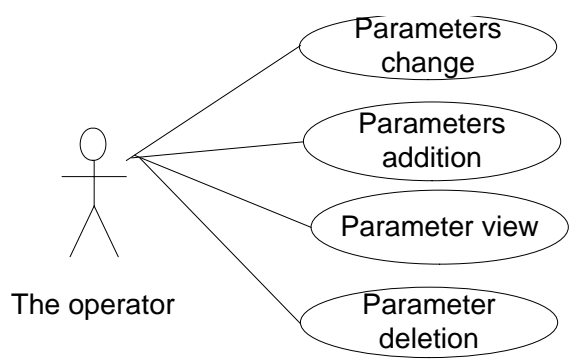

Figure 2 Parameter settings use case diagram

Parameter management is the management of radar equipment parameters. The functions listed in this function module include: parameter addition, parameter modification, parameter viewing and parameter deletion. Figure 2 displays the parameter management use case diagram.

Track management is utilized to track display, track addition, track modification, and manual track admission management. Figure 3 is the track management use case diagram.

Radar management includes a large number of devices so that device management functions is added for this purpose. The functions include device addition, device modification, device check, and device deletion. Figure 4 is the device management use case diagram.

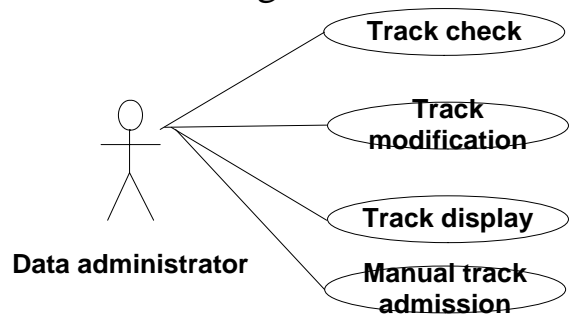

Figure 3 Track management use case diagram

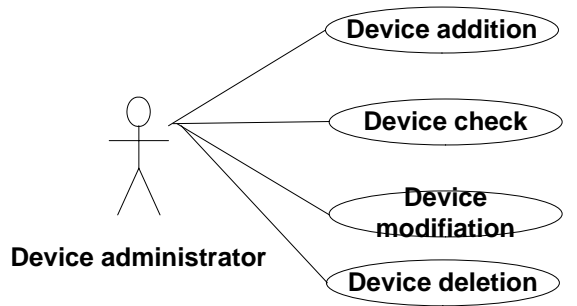

Figure 4 Device management use case diagram

\section{Functional Design of System}

Radar equipment software development was based on $\mathrm{C}++$ and $\mathrm{C \#}$. Among them $\mathrm{C}++$ is used for serial communication development and data exchange management development of the equipment while C\# for system interface development, which improves the development efficiency. In the progress of radar software development, it is designed according to a three-tier architecture: interface layer (view layer), control layer, and data layer. Figure 5 shows system architecture design.

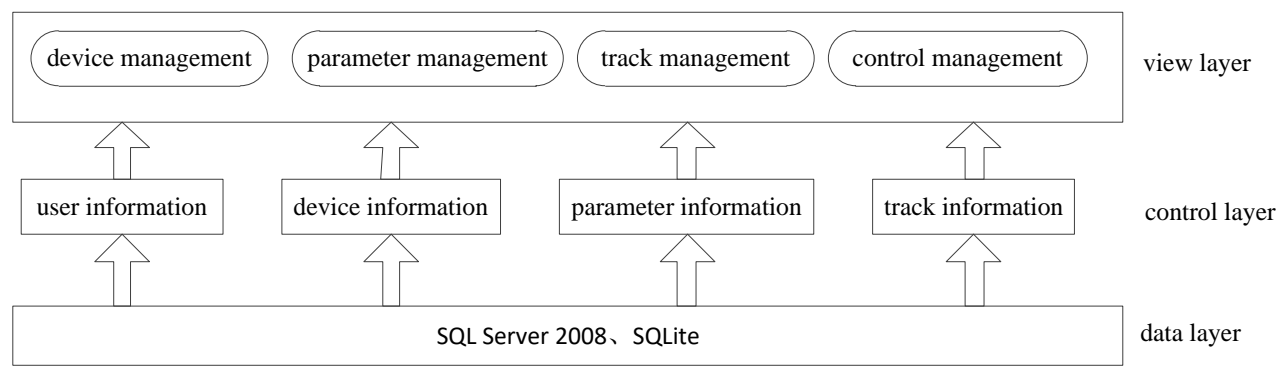

Figure 5 System architecture diagram 
Based on Figure 5, the functions in the interface layer include device management, parameter management, track management and control management. Interface of this layer can be beautified using Component Factory. Krypton. Toolkit. The functions in the control layer are user information, device information, parameter information and trajectory information. SQLServer2008 and open source SQLite database are mainly used in the data layer mainly for data storage management.

Remote control is utilized to remotely operate the radar, whose functions include remote shutdown, remote restart, remote standby. Those functions shall communicate with the device and current communications include serial communication and TCP protocol communication. Serial communication is appropriate for short-distance transmission and TCP protocol for data transmission. The remote control contain Serial_port, TCP and control class. Methods included in the Serial_port class are: panelist () and po-faced (), and they are applied for port addition and port query. Methods included in the TCP class are TCP_insrt () and TCP_find (), which is utilized to protocol addition and protocol checking. Methods included in the control class are Reboot (), Stanby() and Shutdown(), which are applied in remote shutdown, remote restart and remote standby, respectively.

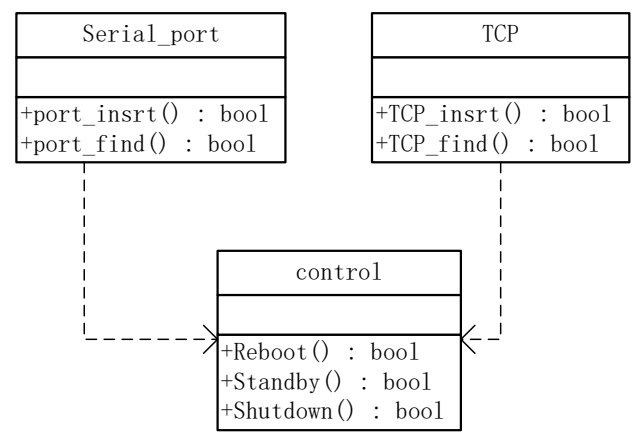

Figure 6 Remote control management class diagram

The functions listed in the parameter setting management include parameter addition, parameter modification, parameter checking and parameter deletion. Figure 7 displays the parameter setting management class. The classes involved are parameter, parameterDALL, parameterBLL and parameterMoble. Methods included in the parameter class are parameter_select(), parameter_insert(), parameter_update() and parameter_delete(), which are usedin parameter viewing, parameter addition, parameter modification and parameter deletion.

The database is utilized to store system-related parameters. The radar software contains data tables with parameter information tables and equipment information tables. The following is a partial data sheet description.

(1) Parameter information table. The table contains fields: parameter number, parameter name, parameter type, content. Table 1 is a detailed description.

Table 1 Parameter information table

\begin{tabular}{|c|c|c|c|c|c|}
\hline Field Name & Type & $\begin{array}{l}\text { Field } \\
\text { Length }\end{array}$ & $\begin{array}{l}\text { Allow } \\
\text { Empty }\end{array}$ & $\begin{array}{l}\text { Is It } \\
\text { Primary }\end{array}$ & $\begin{array}{l}\text { Field } \\
\text { Description }\end{array}$ \\
\hline Radar_paracode & Varchar & 40 & NO & YES & $\begin{array}{l}\text { Parameter } \\
\text { Number }\end{array}$ \\
\hline Radar_paraname & Varchar & 50 & NO & & $\begin{array}{l}\text { Parameter } \\
\text { Name }\end{array}$ \\
\hline Radar_paratype & Varchar & 50 & NO & & $\begin{array}{l}\text { Parameter } \\
\text { Type }\end{array}$ \\
\hline Radar_paracontent & Varchar & 20 & NO & & $\begin{array}{l}\text { Parameter } \\
\text { Content }\end{array}$ \\
\hline Radar_paramark & varchar & 50 & NO & & Note \\
\hline
\end{tabular}

(2) Equipment information table. This table is utilized to store device information. The fields presented in this table are: device number, device name, device type, device usage, acquisition time, price, and so on. Table 2 details the device information table. 
Table 2 Equipment Information Table

\begin{tabular}{|c|c|c|c|c|c|}
\hline Field Name & Type & $\begin{array}{l}\text { Field } \\
\text { Length }\end{array}$ & $\begin{array}{l}\text { Allow } \\
\text { Empty }\end{array}$ & $\begin{array}{l}\text { Is It } \\
\text { Primary }\end{array}$ & $\begin{array}{l}\text { Field } \\
\text { Description }\end{array}$ \\
\hline Radar_eqcode & Varchar & 40 & NO & YES & $\begin{array}{l}\text { Parameter } \\
\text { Number }\end{array}$ \\
\hline Radar_eqname & Varchar & 50 & $\mathrm{NO}$ & & $\begin{array}{l}\text { Parameter } \\
\text { Name }\end{array}$ \\
\hline Radar_eqtype & Varchar & 50 & NO & & $\begin{array}{l}\text { Parameter } \\
\text { Type }\end{array}$ \\
\hline Radar_equse & Varchar & 20 & NO & & $\begin{array}{l}\text { Equipment } \\
\text { Use }\end{array}$ \\
\hline Radar_eqtime & date & & NO & & $\begin{array}{l}\text { Purchase } \\
\text { Time }\end{array}$ \\
\hline Radar_eqcharge & float & & NO & & Price \\
\hline
\end{tabular}

\section{Conclusion}

UML modeling technology was used to analyze the radar software functional requirements. The results showed that the radar software contains the following functions: remote control, parameter setting, track management, and device management. Radar software functions are developed using UML technology class diagram design. Radar software storage database selects SQLServer2008 and open source SQLite database. Through system functions requirement analysis and system functional design, it is ready for the subsequent system development.

\section{References}

[1] Xia Houshi, Ma Min, Wang Houjun. Research on Modeling Method of Radar Test System Based on UML and Petri Net[J]. Journal of Instrumentation, 2009, 30(1) :7-13.

[2] Li Hai. Radar software design built on real-time UML[J]. Modern Radar, 2006, 28(8):47-49.

[3] Xiong Wei, Gao Xia, Wang Li, et al. Modeling and Simulation of UML-based Airborne Radar Simulator System[J]. Systems Engineering and Electronics, 2015, 37(7): 1536-1542.

[4] He Lei, Zhu Ning, Lei Yonglin, et al. Modeling of airborne radar based on UML[J]. Journal of System Simulation, 2014, 26(5): 981-985.

[5] Chen Jie, Wang Lei, Cao Jianyu, et al. Research and Implementation of Radar Simulation System built on Message Center Communication[J]. Journal of System Simulation, 2013, 25 (1) :54-60. 